\title{
$\mathrm{H}$
}

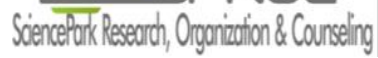

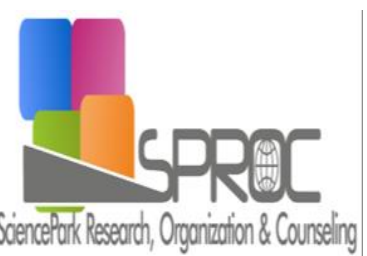

C
$\mathrm{H}$
E
C
$\mathrm{BE}$
A

\section{Long-term supplier selection problem: A case study}

Ercan Senyigit ${ }^{a} * D$

Ismet Soylemez D

Ugur Atici ${ }^{c} \mathrm{G}$
E

D
A C

Suggested Citation:

$\mathrm{E}$

A

Issues Proceedings on Humanities and Social Sciences.

New Trends and

A

D Ç $B \quad G$

C

A

Abstract

G

A

A

\footnotetext{
* ADD E F C E DE CE Ercan Senyigit, D 


\section{Introduction}

C

H D B

H D

C

B

D

C

D B

2. Literature review

$\mathrm{AH} \quad \mathrm{A}$

E

$\mathrm{H}$

$\mathrm{F} \mathrm{AH}$
$\mathrm{H}$

E HEE

G

B

A

B

B

C

A

$\mathrm{H}$

\section{E}


A

D

B
$\mathrm{H}$
C
H D

C

E

\section{Problem statements}

\section{Nomenclature}
I Suppliers
J Criteria
$X_{i j} \quad j$.criteria value of the supplier $i$
$\mathrm{W}_{\mathrm{ij}} \quad$ j.criteria weight of weight of the supplier $\mathrm{i}$
$\mathrm{Y}_{\mathrm{ij}} \quad$ Transformed value of criteria I of supplier $\mathrm{j}$
$\mathrm{G}_{\mathrm{i}} \quad$ Goodness score of supplier i

$$
\operatorname{Max} S_{i}=\sum_{j=1}^{J} W_{i j} * Y_{i j}
$$

s.t.

$$
\begin{aligned}
& w_{i j}-w_{i(j+1)} \geq 0 \quad j=1,2, \ldots,(J-1) \\
& \sum_{j=1}^{J} \mathrm{w}_{i j}=1 \\
& w_{i j} \geq 0 \quad j=1,2, \ldots, 5
\end{aligned}
$$

$$
Y_{i j}=\frac{\left(X_{i j}-\operatorname{Min}_{i=1,2, \ldots,\left\{X_{i j}\right\}}\right)}{\left(\max _{i=1,2, \ldots, I\left\{X_{i j}\right\}}-\min _{\mathrm{i}=1,2, \ldots, I\left\{X X_{i j}\right.}\right\}}
$$


D

\section{The case study}

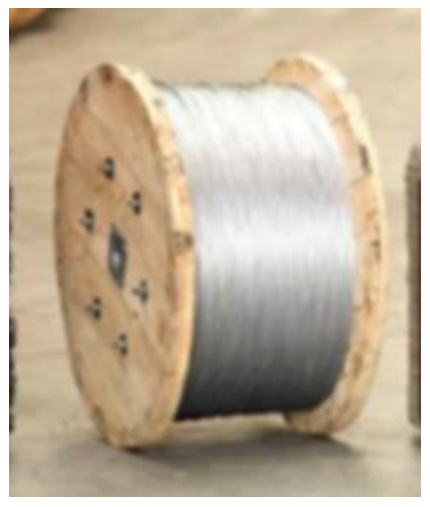

Figure 1. Picture of the pulley.

A

- D DE

- D D

A 
Table 1. Supplier Selection Criteria of the Company and Data Belonging to the Criteria.

\begin{tabular}{lllll}
\hline Suppliers & $\mathbf{Q}(\%)$ & $P(\mathrm{TL})$ & $\mathrm{DE}(\%)$ & $\mathrm{DI}(\mathrm{Km})$ \\
\hline
\end{tabular}

Table 2. Combinations Belonging to Selection Criteria.

\begin{tabular}{|c|c|c|c|c|c|c|c|}
\hline \multirow[t]{2}{*}{ No } & \multicolumn{3}{|c|}{ Combinations } & No & \multicolumn{3}{|c|}{ Combinations } \\
\hline & & $\mathrm{DE}$ & $\mathrm{D}$ & $\mathrm{DE}$ & & $\mathrm{D}$ & \\
\hline & & $\mathrm{D}$ & $\mathrm{DE}$ & $\mathrm{DE}$ & & & D \\
\hline & $\mathrm{DE}$ & & D & $\mathrm{DE}$ & & & D \\
\hline & $\mathrm{DE}$ & D & & $D E$ & & D & \\
\hline & $\mathrm{D}$ & & $\mathrm{DE}$ & $\mathrm{DE}$ & D & & \\
\hline & $\mathrm{D}$ & $\mathrm{DE}$ & & $\mathrm{DE}$ & $\mathrm{D}$ & & \\
\hline & & $\mathrm{DE}$ & D & D & & & $\mathrm{DE}$ \\
\hline & & D & $\mathrm{DE}$ & D & & $\mathrm{DE}$ & \\
\hline & $\mathrm{DE}$ & & D & $\mathrm{D}$ & & & $\mathrm{DE}$ \\
\hline & $\mathrm{DE}$ & D & & D & & $\mathrm{DE}$ & \\
\hline & $\mathrm{D}$ & & $\mathrm{DE}$ & $\mathrm{D}$ & $\mathrm{DE}$ & & \\
\hline & $\mathrm{D}$ & $\mathrm{DE}$ & & D & $\mathrm{DE}$ & & \\
\hline
\end{tabular}

Table 3. Normalized Data.

\begin{tabular}{lllll}
\hline Suppliers & Q & P & DE & DI \\
\hline
\end{tabular}

\section{Modeling and solution}




$$
\begin{gathered}
\operatorname{Max} G_{\mathrm{i}}=w_{\mathrm{i} 1} Q+w_{\mathrm{i} 2} P+w_{\mathrm{ia}} D E+w_{i 4} D I \\
\text { St. } \\
w_{\mathrm{i} 1}-w_{\mathrm{i} 2} \geq 0 \\
w_{\mathrm{i} 2}-w_{\mathrm{ia}} \geq 0 \\
w_{\mathrm{i} a}-w_{\mathrm{i} 4} \geq 0 \\
w_{\mathrm{i} 1}+w_{\mathrm{i} 2}+w_{\mathrm{ia}}+w_{\mathrm{i} 4}=1 \\
w_{\mathrm{i} j} \geq 0
\end{gathered}
$$

\section{Modeling and solution}

A

$\mathrm{H}$

A

A 
Table 4. Results

\begin{tabular}{|c|c|c|c|c|c|c|c|c|c|c|}
\hline \multirow[t]{2}{*}{ Ranking No } & \multicolumn{4}{|c|}{ Ranking of criteria } & \multirow[t]{2}{*}{ S1 } & \multirow{2}{*}{\begin{tabular}{|l|} 
S2 \\
1.00
\end{tabular}} & \multirow[t]{2}{*}{ S3 } & \multirow[t]{2}{*}{ S4 } & \multirow[t]{2}{*}{ S5 } & \multirow[t]{2}{*}{ Ranking of suppliers } \\
\hline & & & $\mathrm{DE}$ & $\mathrm{D}$ & & & & & & \\
\hline & & & D & $\mathrm{DE}$ & & 1.00 & & & & \\
\hline & & $\mathrm{DE}$ & & D & & 1.00 & & & & \\
\hline & & $\mathrm{DE}$ & $\mathrm{D}$ & & & 1.00 & & & & \\
\hline & & D & & $\mathrm{DE}$ & & 1.00 & & & & \\
\hline & & D & $\mathrm{DE}$ & & & 1.00 & & & & \\
\hline & & & $\mathrm{DE}$ & D & & & & & 1.00 & \\
\hline & & & $D$ & $\mathrm{DE}$ & & & & & 1.00 & \\
\hline & & $\mathrm{DE}$ & & D & & & & & 1.00 & \\
\hline & & $\mathrm{DE}$ & D & & & & & & 1.00 & \\
\hline & & D & & $\mathrm{DE}$ & & & & & 1.00 & \\
\hline & & D & $\mathrm{DE}$ & & & & & & 1.00 & \\
\hline & $\mathrm{DE}$ & & D & & & & & 1.00 & & \\
\hline & $\mathrm{DE}$ & & & D & & & & 1.00 & & \\
\hline & $\mathrm{DE}$ & & & D & & & & 1.00 & & \\
\hline & $\mathrm{DE}$ & & D & & & & & 1.00 & & \\
\hline & $\mathrm{DE}$ & D & & & & & & 1.00 & & \\
\hline & $\mathrm{DE}$ & $D$ & & & & & & 1.00 & & \\
\hline & D & & & $\mathrm{DE}$ & & & & 1.00 & & \\
\hline & $\mathrm{D}$ & & $\mathrm{DE}$ & & & & & 1.00 & & \\
\hline & D & & & $\mathrm{DE}$ & & & & 1.00 & & \\
\hline & $\mathrm{D}$ & & $\mathrm{DE}$ & & & & & 1.00 & & \\
\hline & $\mathrm{D}$ & $\mathrm{DE}$ & & & & & & 1.00 & & \\
\hline & $\mathrm{D}$ & $\mathrm{DE}$ & & & & & & 1.00 & & \\
\hline & & A & & & & & & 0.92 & & \\
\hline
\end{tabular}

\section{References}

B C

Operations Research, 32

Computers \&

C

A

C

C $\mathrm{H}$ 55 ,
$\mathrm{E}$ $\mathrm{E}$ International Journal of Production Economics, 166 
